sungswidrig erklärt werden sollte, dann kann das dazu führen, daß sich hinfort politisch bewußte Arbeiter gegenüber einem so ausgelegten Grundgesetz auf Legitimität gegen Legalität berufen.

Jürgen Seifert

\title{
Soziale Isolation und sensorische Deprivation
}

Die Tatsache konstatieren, daß in der BRD zahllose Gefangene Haftbedingungen ausgesetzt sind, die einer totalen Isolation gleichkommen, und diese Tatsache Folter nennen, bedarf der Erläuterung, zunächst des Begriffs und dann der Folgen.

$\mathrm{Da}$ isoliert wird, ist unbestritten, "aus Sicherheitsgründen, um die Ermittlungen oder die Ordnung der Anstalt nicht zu gefährden. "Konkret bedeutet das für den Gefangenen, daß tendenziell jeder soziale Kontakt nach draußen und im Innern der Anstalt unterbunden wird (soziale Isolierung) und, in der Konsequenz dieser Maßnahmen, ein fast vollständiges Aushungern der Seh-, Hör-, Riech-, Geschmacks- und Tastorgane (sensorische Deprivation).

Bei den zuständigen Stellen scheint sich der Eindruck zu verfestigen, daß solche Sicherheits-, Ermittlungs- oder Ordnungsgründe künftig verstärkt in Betracht kommen: in Frankfurt/M.-Preungesheim wurde Anfang Juni 73 ein Untersuchungsgefängnis in Betrieb genommen, das Isolation systematisiert und baulich für alle Gefangenen möglich macht. Bekannt geworden sind die Betonmauer - Sichtblenden vor jedem Zellenfenster. Dabei wird der Verdacht, daß es sich um geplante und systematisierte Maßnahmen handelt, noch erhärtet durch eine andere Tatsache: die zumindest in Europa perfektionierteste und intensivierteste Forschung zum Thema Isolation und Deprivation wird derzeit im Laboratorium für klinische Verhaltensforschung im Universitätskrankenhaus Eppendorf der Universität Hamburg durchgeführt. Dort gibt es einen mit großem technischen Aufwand hergestellten, absolut "stillen Raum ", in dem Versuchspersonen derart isoliert und depriviert werden können, daß selbst im Raum erzeugte Geräusche nicht wahrgenommen werden können. Die Zeichnung, die der Beschreibung $^{1}$ dieser "camera silens « beigefügt ist, mutet schon an, als sei sie den Akten eines modernen Frankenstein entnommen, und stolz berichten die Erfinder vom schwingungsfrei aufgehängten "Raum im Raum», von den 12 Hi-Fi (High Fidelity) Lautsprechern in Rundumanordnung, durch die der Raum beschallt werden kann, von der Infrarot-Fernsehkamera, mit deren Hilfe die "Vp« ständig beobachtet, von Richtmikrophon und Förstersonde, mit deren Hilfe jede Bewegung und Lageveränderung registriert werden kann. Vollständigkeit aber wird erst erreicht sein, »wenn unsere polyphysiographische Ausrüstung mit telemetrischer Datenübermittlung in Benutzung genommen wird (16 Kanäle). Vorgesehen sind multiple EEG-Ableitungen, EKG, PGR. ERG- und AtemfrequenzRegistrierung. «² Dazu noch ein Gerät zur Aufzeichnung von Magenmotilität.

Diesem Perfektionismus der Forschungsmethoden steht die scheinbare Gleichgültigkeit und Naivität der Forscher bezüglich des Forschungszwecks gegenüber. Auf Anfrage läßt der Erfinder Prof. Dr. med. Jan Gross seinen Mitarbeiter

1 J. Gross, J. M. Burchard und P. Kempe, Sensorische Deprivation, Psychiat. Neurol. Neurochir., 73 (1970), Amsterdam.

2 Ebenda S. I 91. 
Dipl. Psych. P. Kempe mitteilen, *bisher handele es sich "ausschließlich um Grundlagenforschung, wobei die in unserem Laboratorium erzielten Bedingungen als Modellsituation für andere, unter bestimmten Bedingungen auch im Alltag realisierte Lebensbedingungen dienen können. « ${ }^{3}$ (Hervorhebungen vom Verfasser), und beschwichtigend fügt er hinzu, »daß mögliche spätere Ergebnisse unserer Arbeitsgruppe wohl eher dazu angetan sein würden, solche Faktoren des Strafvollzugs (Isolation, d. Verf.) zu eliminieren. « ${ }^{4}$

Es fällt schwer, derlei $Z$ weckfreiheit unbesehen hinzunehmen, zumal dann, wenn man auf besonders exotische Nebengeleise geführt wird. In einem Aufsatz ${ }^{5}$ rekonstruieren die beiden genannten Forscher den Ausgangspunkt ihrer wissenschaftlichen Bemühungen: die Einsamkeit des Einhandseglers bei der Reise um die Welt (finanziert von der Deutschen Forschungsgemeinschaft!?). Darin stellen sie eindeutig fest, daß die "soziale Isolation ... einer der Hauptfaktoren (ist), welcher die psychische Lage des Einhandseglers ausmadht. $\aleph^{6}$

Diese Lage ist gekennzeichnet durch folgende belastende Bedingungen:

- Soziale Isolation

- Schlafdefizit

- Angst und Anspannung

- Sensorische und Perzeptive Deprivation

- Nahrungsmonotonisierung oder Mangel

- Sexuelle Frustration

- Bewegungseinschränkung?

Es bedarf keiner besonderen Scharfsinnigkeit, um festzustellen, daß dies auch die Bedingungen sind, denen Gefangene in unseren Haftanstalten unterworfen sind oder jederzeit unterworfen werden können.

Bereits die unsystematischen Berichte solcher Einhand-Weltumsegler geben Aufschluß über die Folgen der Isolation:

- Verlangsamung der Denkfähigkeit

(korreliert mit der Dauer der sozialen Isolation)

- Allgemeiner Motivationsverlust (!)

- Ausschaltung der Selbsterhaltungs- bzw. Selbstbehauptungsmechanismen (!)

- Psychosomatische Störungen (Magengeschwüre)

- (meist visuelle) Halluzinationen und Wahnvorstellungen ${ }^{8}$

Um diese Beobachtungen wissenschaftlich kontrolliert verifizieren zu können, mußten Situationen konstruiert werden, die es ermöglichen, den Wirkungszusammenhang solcher Bedingungen wie Umgebungsfaktoren und Isolationsdauer einerseits, Persönlichkeitsmerkmalen der Versuchsperson, etwa größere Widerstandsfähigkeit und sozialer Status, sowie das Verhältnis zwischen dem Versuchsleiter und der Versuchsperson in Bezug zu setzen zu Verhaltensänderungen in und nach dem Versuch: Durchhaltedauer, Wahrnehmungs- und Verhaltensveränderungen, emotionale Anpassung sowie physiologische Veränderungen.

Nun stellt sich die Frage, warum wurde dann mit der »camera silens « eine Versuchsanordnung hergestellt, die über die soziale Isolation hinaus eine totale sensorische Deprivation erlaubt. Die Antwort gibt Gross in einem Aufsatz zusam-

3 Persönlidhes Anschreiben.

${ }^{4}$ Ebda.

5 P. Kempe und J. Gross, Psychologische Aspekte von Einhand-Weltumsegelungen, "Die Yachte S, S. 67, März (1970).

Ebda.

7 Ebda.

8 Ebda. 
men mit L. Svab ${ }^{\theta}$ mit dem er bis r 968 das Psychiatrische Forschungsinstitut Prag betrieb. In diesem Aufsatz heißt es: „Da die Einschränkung aller äußeren Reize auch die soziale Stimulation betrifft, und so in jeder sensoriellen Deprivation auch implizit eine soziale Isolation enthalten ist, bringt die Auswertung der Elemente der sozialen Isolation in der sensoriellen Deprivation verschiedene methodologische Hindernisse mit sich. «10 Diese Schwierigkeit, nicht auseinanderhalten zu können, sollte die Versuchsanordnung eigentlich hinfällig machen, wenn nicht die sensorische Deprivation eine andere bemerkenswerte Folge hätte: "Unserer Meinung nach kann auch die erböbte Suggestibilität der Versuchsperson als eine spezifische Folge der Deprivations-Situation angesehen sein, da in dieser Situation ihre Fähigkeit, die umgebende Realität zu testen, herabgesetzt oder gar blockiert ist (Goldenberger u. Holt). Dies macht sie in erhöhtem Maße von Informationen, die ihr durch den Experimentator vermittelt werden, abhängig. Diese vorwiegend einseitige Abhängigkeit der Versuchsperson vom Experimentator ist in der sensoriellen Deprivation ausgeprägter als in anderen experimentellen Situationen..." (Hervorhebungen vom Verf.) ${ }^{11}$ Im Klartext heißt das, in der "camera silens « lassen sich im kurzfristigen Experiment psychische Zustände auslösen, die sonst nur bei längerfristiger sozialer Isolation beobachtet werden können. Die deprivierte Situation versetzt in psychischen und physischen Stress, macht Angst und Wut, weil dem ohnmächtigen Objekt des Versuchs die Orientierungs- und Regulationskriterien entzogen sind, die ihm die aktive Gestaltung der Situation erlaubten. Die Folge ist Desorientierung und Desorganisation des Verhaltens.

In der Realität bietet das Gefängnis die Möglichkeit, Menschen derartigen Bedingungen auszusetzen, wobei an die Stelle der totalen sensoriellen Deprivation die Wirkung der Dauerisolation tritt. Allerdings nimmt im Gefängnis den Platz des Experimentators für Grundlagenforschung der Inquisitor ein, dem es um Geständnisse geht. Der Blick in ein beliebiges Nachschlagewerk der Psychiatrie überzeugt davon, daß der Zusammenhang in der Branche durchaus gesehen wird: "Die Anwendung von Isolierung und Furcht als Komponenten der komplexen Prozeduren, durch die Menschen dazu gebracht werden, Verbrechen zu gestehen, an denen sie unschuldig sind, bezeugt unser machtvolles Bedürfnis nach Stabilität und Anerkennung mittels sozialer Interaktionen; selbst Bestrafung und Zurückweisung werden unter Umständen der Isolierung vorgezogen. «12 Auch das in Klartext übersetzt: die Psychiatrie stellt subtile Methoden bereit, die grobe und mit dem »demokratischen Selbstverständnis « unvereinbare körperliche Folterungen zur Erzwingung von Geständnissen und Brechung von Persönlichkeiten ersetzen können. Dabei müssen diese Methoden Folter genannt werden, nicht nur, weil sie den gleichen Zweck erfüllen, nur lautloser, sondern auch, weil sie ebenso nachhaltige Verstümmelungen des Opfers zur Folge haben, nur weniger sichtbar. In der psychiatrischen Literatur ist völlig unbestritten, „daß Isolierung in Verbindung mit extremem physischem und psychischem Streß (große Angst) psychoseartige Reaktionen hervorrufen kann. «13 Wenn ein Probant, der einem 8-Stunden-Experiment gerade entgangen war, »die Versuchsleiter offen

- J. Gross und L. Svab, Die experimentelle sensorielle Deprivation als Modellsituation der psychotherapeutischen Beziehung, Der Nervenarzt 40 . Jg., Heft 1, 1969.

1) Ebda.

11 Ebda.

12 Fredrick C. Redlich und Daniel X. Freedmann, Theorie und Praxis der Psychiatrie, Ffm 1970, S. 815 .

13 Ebda S. 815 . 
anklagte, diese versuchten ihn in den Wahnsinn zu treiben, um ihn dann als Patienten in der Klinik behalten zu können ", ${ }^{14}$ dann sind das ja wohl am allerwenigsten "paranoide Wahnbildungen ", wie die Versuchsleiter interpretieren, sondern da haben 8 Stunden eben nicht ausgereicht, einen irre zu machen. Viel eher hat das Opfer etwas vom Wesen der Psychiatrie erkannt.

Festzuhalten bleibt, daß sich die Haftsituation von der Experimentalsituation in zwei Punkten verschärfend unterscheidet: in sie gerät man unfreiwillig und sie ist noch weniger einschätzbar, weil der anonyme Apparat willkürlich gegen die Persönlichkeit eingesetzt werden kann. Wenn sich bei Experimenten schon nach zwei bis drei Stunden signifikante Wirkungen zeigen, muß man fragen, wie nachhaltig die Schädigungen nach monate- oder jahrelanger Isolation sein werden.

In dem schon zitierten Aufsatz von Kempe und Gross geben die Autoren den Soloseglern schließlich noch Hinweise, wie den psychischen Folgen der Isolation begegnet werden kann. U. a. nennen sie:

- geistige Aktivität, wie das Lesen interessanter Bücher, das Erlernen fremder Sprachen, Problemlösen und kreatives Denken

- abwechslungsreiche Nahrung mit besonderen Vitaminzusätzen

- Gymnastik

- passive Kontakte (Radio)

- sinnvoller Tagesrhythmus

Ohne die Autoren aus ihrer heilen Grundlagen-Forschungs-Welt aufschrecken zu wollen, muß gesagt werden, daß sich dieser Katalog auch als Handlungsanweisung für Vollzugsbeamte lesen läßt. Alle die genannten Punkte sind im Gefängnis manipulierbar und werden auch entsprechend eingesetzt.

Es gibt Hinweise dafür, daß in absehbarer Zeit auch solche Nischen des Widerstands nachhaltig versperrt werden können: pharmakologisch induzierte Veränderungen der Orientierungsfähigkeit brechen noch schneller, noch lautloser und noch nachhaltiger.

Fassen wir zusammen: In der BRD sind nur sehr notdürftig verschleierte Tendenzen zu beobachten, Gefangene systematisch zu isolieren und damit Widerstand und Willen zu brechen. Das geht nicht ab ohne nachhaltige psychische und physische Dauerschäden bei den Betroffenen und kann deshalb nur als Folter bezeichnet werden.

$\mathrm{Da}$ gerade in der BRD in großem Stil Forschung in dieser Richtung getrieben wird, scheinbar ohne daß die beteiligten Wissenschaftler sich um die Anwendung ihrer Ergebnisse kümmerten, kann nicht länger nur als Streit um die Verantwortlichkeiten von Wissenschaft aufgefaßt werden und als wissenschaftstheoretisches Problem der Psychiatrie diskutiert werden. Der holländische Psychiater Sjef Teuns ${ }^{15}$ hat seine deutschen Kollegen gemahnt: "Nicht der Kapo, der die Knöpfe des vorfabrizierten Folterinstruments bedient, ist der Hauptschuldige im neuzeitlichen Foltersystem, sondern diejenigen, die in Kenntnis der Zusammenhänge Grundlagenforschung betreiben, aus der die Methodik des Systems entwickelt wird und hervorgeht.*

Frank Olaf Radtke

14 J. Gross, P. Kempe, Ch. C. Reimer, Wahn bei sensorischer Deprivation und Isolierung, vervielfältigtes Manuskript der Universitätsklinik Hamburg, S. 6.

15 S. Teuns, Isolation - Sensorische Deprivation als Foltermethode, Vortrag gehalten am I I. 5. 73 in Frankfurt, vervielfältigtes Manuskript. 